\title{
Investigation of PBGA Solder Joint Failure in Reflow Process
}

$$
\text { Yu GUO }{ }^{1, a} \text {, Zhi-Li SUN }{ }^{1} \text {, Ming-He LIU² and Xiao-Ying MA }{ }^{1}
$$
${ }^{1}$ School of Mechanical Engineering \&Automation, Northeastern University, Shenyang, China,
110819

${ }^{2}$ School of Mechanical Engineering, Shenyang Jianzhu University, Shenyang, China, 110168

Keywords: PBGA package, Solder joint, Reflow process, Stress and strain, Simulation.

\begin{abstract}
In PBGA package, solder joint provides not only mechanical support, electrical conduction and thermal conduction effect, but also withstands frequent thermal, mechanical, electrical loads in the high temperature operation of the electronic device. Thus, solder joint quality is directly related to PGBA products reliability in the practical application. Based on the finite element method, the thesis studies the thermal stress and strain distribution of PBGA solder joint during the reflow process so as to locate the maximum stress and strain. Furthermore, dangerous solder joint of PBGA failure is predicted as well. Studies show that the maximum stress and strain of solder joint are present in the corner joints between BGA and PCB connection surface. From this point of view, the danger joints of structural failure are the corner solder joints of PBGA.
\end{abstract}

\section{Introduction}

Ball grid array package (Ball Grid Array, BGA) is a widely used package currently which refers the ball grid array as I / O terminals at the bottom of the electronic device [1]. BGA package has great feature of small package size, a lot of pin, high reliability, good electrical properties and low cost. The current mainstream products are plastic ball grid array BGA packaging (PBGA) package and ceramic ball grid array (CBGA). Where, PBGA becomes the major consumer electronics package for its lower price $[2,3]$.

As the key process of Printed Circuit Board Assembly (PCBA) production, reflow soldering determines the final quality and reliability of products $[4,5]$. In the reflow process, since thermal parameters of printed circuit board assembly (PCBA) material varies from temperature dependent and there is anisotropous among the elements, solder joints are prone to internal thermal stress and strain. Furthermore, the stress concentration is produced and crack is initiation and propagation, which causes PBGA failure in the application process and leads to decrease of product reliability eventually $[6,7]$. Therefore, the failure and reliability analysis of PBGA is a prerequisite to ensure the quality of the PCBA.

Currently, the judgments of solder crack failure are direct observation and indirect testing as main method. While, the PBGA solder joint size is so small that the direct observation and measurement method requires very precise measuring equipment with higher technical requirements and great investment costs. Therefore, it has important on the numerical simulation of solder cracks for practical engineering [8]. Based on the heat transfer theory, the paper simulates the PBGA transient thermal stress and strain distribution with finite element thermal - structural coupling method. Then potential crack generation region is predicted as well. The study provides a theoretical basis for further evaluation of the PCBA reliability.

\section{Mathematic Model for PBGA Temperature Field}

Generally speaking, temperature field is a function of spatial coordinates and time. Temperature field inside the object is determined by its general equation and thermal differential equations. Besides, reflow process can be considered as a nonlinear transient problem. For thermal properties of material changes with temperature drastically, Its heat conduction equation is described with the general form of three-dimensional unsteady heat conduction differential equations [9]: 


$$
\rho c \frac{\partial t}{\partial \tau}=\frac{\partial t}{\partial x}\left(\lambda \frac{\partial t}{\partial x}\right)+\frac{\partial t}{\partial y}\left(\lambda \frac{\partial t}{\partial y}\right)+\frac{\partial t}{\partial z}\left(\lambda \frac{\partial t}{\partial z}\right)+\Phi
$$

The reflow process is heat exchange between the reflow oven and the components. The main exchanging method is the combined effect of heat convection and radiation. We transfer the radiation heat to convection heat while modeling so as to calculate and load conveniently.

One boundary condition is convective heat transferring. Equation (2) is derived by Newton cooling formula:

$$
q_{\text {conv }}=h_{c}\left(T_{1}-T_{2}\right)
$$

Where, $q_{c o n v}$ is convection heat conduction per unit area; $h_{c}$ is the convective heat transfer coefficient; $T_{1}$ is the absolute temperature within the oven; $T_{2}$ is the absolute temperature on the point of PCBAs.

The heat transfer process can be regarded as the heat transformation from the upper and lower surfaces of the oven to the PCBAs in the middle in some zone [10]. According to reference [11], the heat transfer convection coefficient $h_{\mathrm{c}}$ of a flat plat when air flow along it is:

$$
h_{c}=\frac{0.664 u^{1 / 2}\left(\eta c_{p}\right)^{1 / 3} \lambda^{2 / 3}}{(v L)^{1 / 2}}
$$

Where, $u$ is the flow rate of the gas, in this paper $u=5 \mathrm{~m} / \mathrm{s} ; \eta$ is dynamic viscosity of the gas; $c_{p}$ is heat capacity of the gas; $\lambda$ is kinematic viscosity of the gas: $v$ is fluid viscosity of the gas; $L$ is the length of the PCBAs plate. In this case, heat convection coefficient of hot air $h_{\mathrm{c}}$ can be calculated according to formula (3).

During the reflow process, the other boundary condition is radiation. According to the formula for thermal radiation heat transfer:

$$
Q=\frac{\sigma\left(T_{1}^{4}-T_{2}^{4}\right)}{\frac{1-\varepsilon_{1}}{\varepsilon_{1} A_{1}}+\frac{1-\varepsilon_{2}}{\varepsilon_{2} A_{2}}+\frac{1}{A_{2} X_{2.1}}}
$$

Where, $\sigma$ is the blackbody radiation constant; $\varepsilon_{1}, \varepsilon_{2}$ are the emission coefficient of a certain point on the oven chamber and on the PCBAs respectively; $A_{1}, A_{2}$ are the area of the two heat transfer surfaces; $X_{1.2}$ is angular coefficient of surface 1 and 2. According to reference [9], simplify the heat transfer equation per unit area as follows:

$$
q_{\text {rad }}=\frac{\sigma\left(T_{1}^{4}-T_{2}^{4}\right)}{1 / \varepsilon_{1}+1 / \varepsilon_{2}-1}
$$

Where, $\mathrm{q}_{\mathrm{rad}}$ is radiation heat conduction per unit area; $T_{1}$ and $T_{2}$ are the absolute temperature of the oven and some point on PCBAs respectively. In order to facilitate the engineering calculations, the radiation heat transfer is usually converted to the convective heat. Thus, the heat conduction formula can be written into the form as Newtonian cooling formula $q_{r a n d}=h_{r}\left(t_{1}-t_{2}\right)$. So the radiation heat transfer coefficient which converted to form of convective heat transfer coefficient $h_{r}$ is

$$
h_{r}=\frac{\sigma \varepsilon_{1} \varepsilon_{2}\left(T_{1}^{2}+T_{2}^{2}\right)\left(T_{1}+T_{2}\right)}{\varepsilon_{1}+\varepsilon_{2}-\varepsilon_{1} \varepsilon_{2}}
$$

Combine these heat transfer methods, and set $h=h_{c}+h_{r}$. Then the boundary load is

$$
q=h\left(t_{\mathrm{w}}-t_{\mathrm{f}}\right)
$$

Where, $q$ is all the heat applied to unit volume from boundary; $h$ expresses convective heat transfer coefficient when convection and radiation superimposed. 


\section{Finite Simulation for PBGA solder}

\section{PBGA Finite Element Model}

A PBGA in the PCBA is chosen as study object randomly. Taking the geometry and failure form of solder joint into account, geometry of PBGA needs precise modeling so as to make the simulation results of heat stress and strain field more accurate. PBGA package includes balls, chips, BT substrate and the plastic body and other structures. PBGA solder joints were arrayed peripherally below the device with a total of $14 \times 14$ spot welds. Since the selected model appears an axisymmetrical structure, only a quarter of PBGA and its Printed Circuit Board (PCB) is selected for modeling. Thermal property parameters of PBGA are shown in table 1.

Table 1. Thermal properties parameters

\begin{tabular}{|c|c|c|c|}
\hline Part & $\begin{array}{c}\text { Elastic Modulus } \\
(\mathrm{Gpa})\end{array}$ & $\begin{array}{c}\text { Thermal expansion } \\
\text { coefficient }\left(10^{-6} /{ }^{\circ} \mathrm{C}\right)\end{array}$ & $\begin{array}{c}\text { Poisson's ratio } \\
v\end{array}$ \\
\hline PCB & 27 & 15 & 0.39 \\
\hline Solder joint & $60.73-0.0574 T$ & $16.66+0.017 T$ & 0.36 \\
\hline Chip & 167 & 2.7 & 0.3 \\
\hline BT substrate & 17.8 & 15 & 0.39 \\
\hline Plastic BGA & 13 & 15 & 0.3 \\
\hline
\end{tabular}

Finite element model of PBGA solder joint is shown in figure 1.

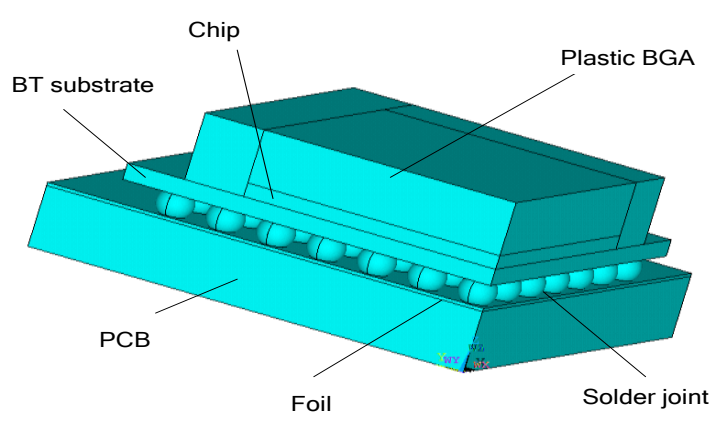

Figure 1. PBGA model

\section{Boundary Conditions and Loading}

A 10 zones reflow oven is used as example for simulation. Then convective heat transfer coefficient is loaded according to the time PCBA going into the oven zones correspondingly. The length of each oven zone is $500 \mathrm{~mm}$. The belt speed is $850 \mathrm{~mm} / \mathrm{min}$. Table 2 lists the setting temperature of each zone.

Table 2. Setting parameters of oven zones

\begin{tabular}{|c|c|}
\hline Oven zone & Setting temperature $/{ }^{\circ} \mathrm{C}$ \\
\hline$\# 1$ & 160 \\
\hline$\# 2$ & 165 \\
\hline$\# 3$ & 180 \\
\hline$\# 4$ & 185 \\
\hline$\# 5$ & 205 \\
\hline$\# 6$ & 240 \\
\hline$\# 7$ & 260 \\
\hline$\# 8$ & 230 \\
\hline$\# 9$ & 60 \\
\hline$\# 10$ & 20 \\
\hline
\end{tabular}


Since the just a quarter of the solid model is analyzed during simulation, symmetry constraint need to be imposed on the newly cutting section. Besides, in order to prevent movement in the overall model analysis, full constraints needs to be imposed on the center of the solid model. Boundary condition of PBGA model is shown in figure 2. Temperature loading on PCBA in each zone is controlled by * DO loop of APDL language in ANSYS.

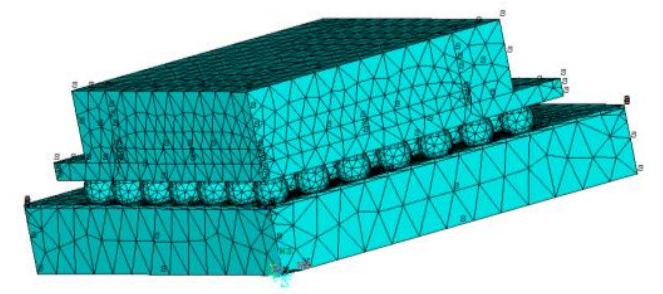

Figure 2. Boundary condition of PBGA model

\section{Simulation Results and Discussion}

It is known from figure 3 that PBGA suffers significant thermal loads during the reflow process. This is mainly due to the anisotropy of different parts, some degree of expansion and contraction occurs on the PCBA. While the deformation coordination requirements of internal or external constraints makes PCBA cannot deformed freely, which led to heating stress produced in each part of PCBA.

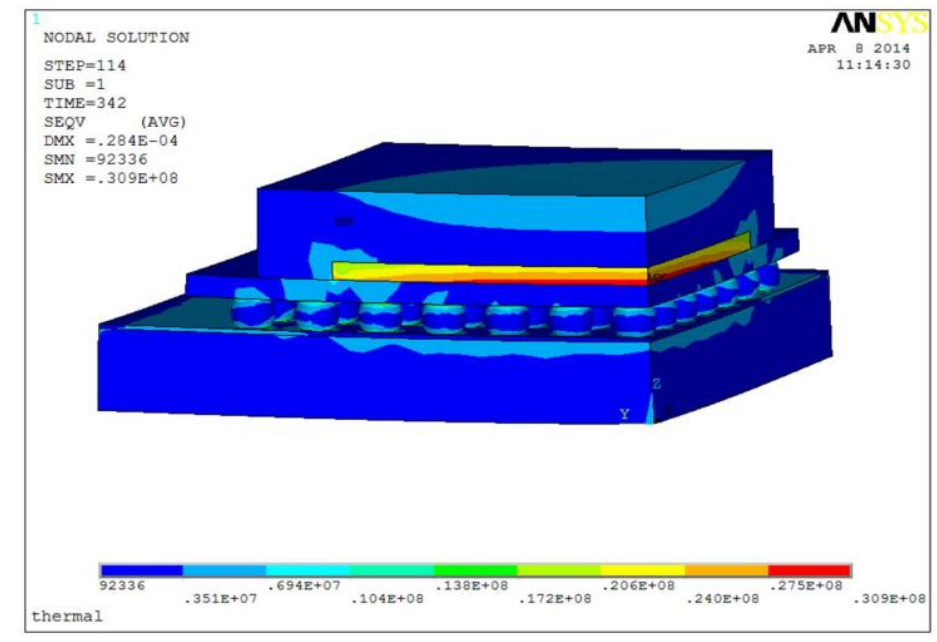

Figure 3. Stress of PCBA

Figure 3 also shows that thermal stress is mainly concentrated at upper and lower end faces of the solder joints, and the thermal stress of intermediate portion is small. This is mainly caused by mismatched thermal expansion coefficients of PCBA components. When the temperature rises, the joints will expanse. However, the greater rigidity of PBGA plays an inhibitory effect on the free expansion joints. Therefore, compressive stress is produced on the contact surface between solder and PBGA. Besides, Thermal expansion coefficient of PCB is less than that of solder joints. A certain warpage on PCB may produce during the reflow process, which inhibits the expansion joints as well. Therefore, the thermal stress of the solder joints is mainly produced in the position of the end face of the solder joint.

From figure 4, it is obvious that Thermal stress of solder joint in the middle part is at the minimal value. The closer to the edge of the BT-substrate, the greater of the joint thermal stress is. The range of stress effect is enlarged as well. The maximum stress reaches 25.1MPa. Figure 5 shows a similar trend with figure 4, whose maximum stress reaches 37.1MPa. Both the Maximum stresses are 
present in corner position between the joints connecting surface and PBGA and the BT-substrate. The cause of this thermal stress distribution is mainly due to the PBGA solder joint from the outside is far from the intermediate position than the inside of the solder components. In the heat loading process, the mismatch coefficient of thermal expansion of the components results in a certain degree of PCB warpage. But the amount of deformation in the central part is smaller than that of the end face. Thus the outer solder joints withstand the maximum stress.

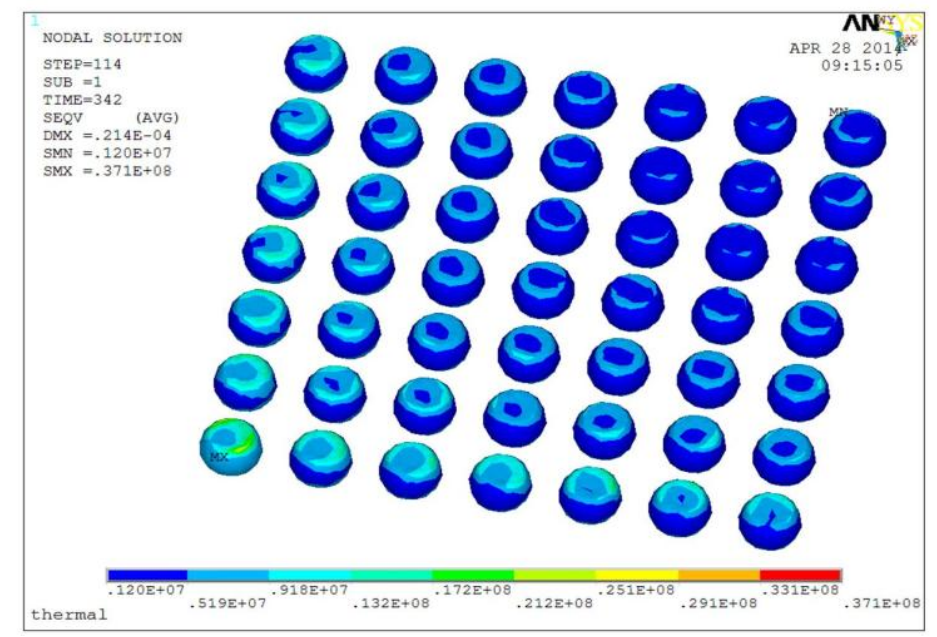

Figure 4. Stress of solder paste contact with copper

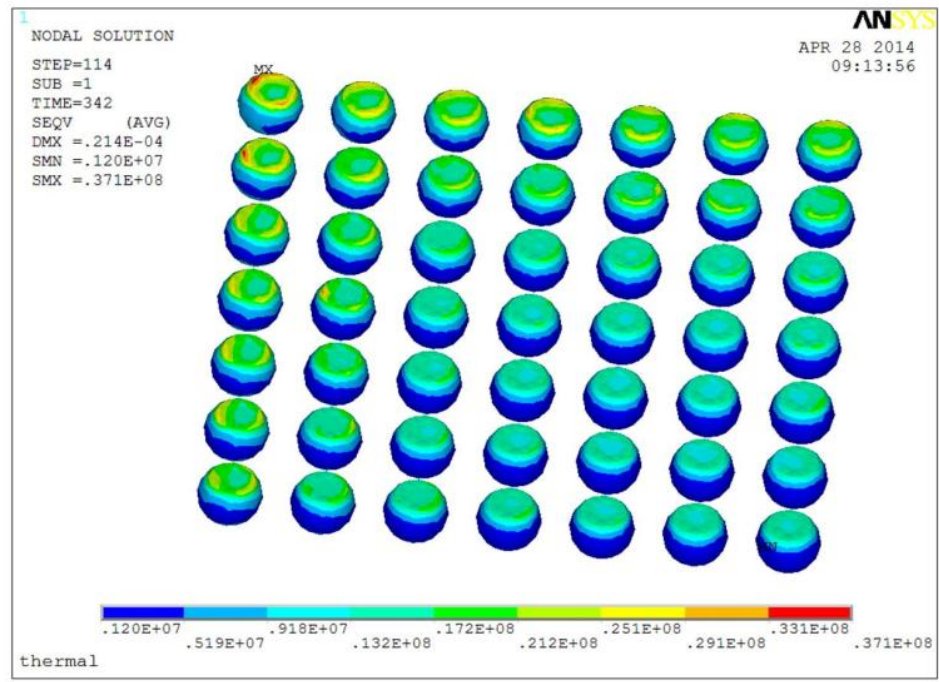

Figure 5. Stress of solder paste contact with BT substrate

When the thermal load acts on PCBA, thermal stress and strain have greater impacts on the joints failure. Therefore study on thermal load PCBA joint quality control and failure analysis has practical significance. From figure 6, strain distribution of PCBA appears smaller in the center portion and greater at the edges. It is seen from figure 7 that four solders located in the center of PBGA presents the minimal thermal deformation. With the pads gradually away from the center, the thermal deformation is also growing. It is due to the whole PCBA is an axisymmetric geometry. Deformation at the center portion of PCBA does not occur along the $X, Y$ direction during the reflow process. While thermal deformation may continue to accumulate with the portion away from the center position until the edges. So the edge of PBGA researches the maximum thermal deformation of $21.4 \mu \mathrm{m}$. 


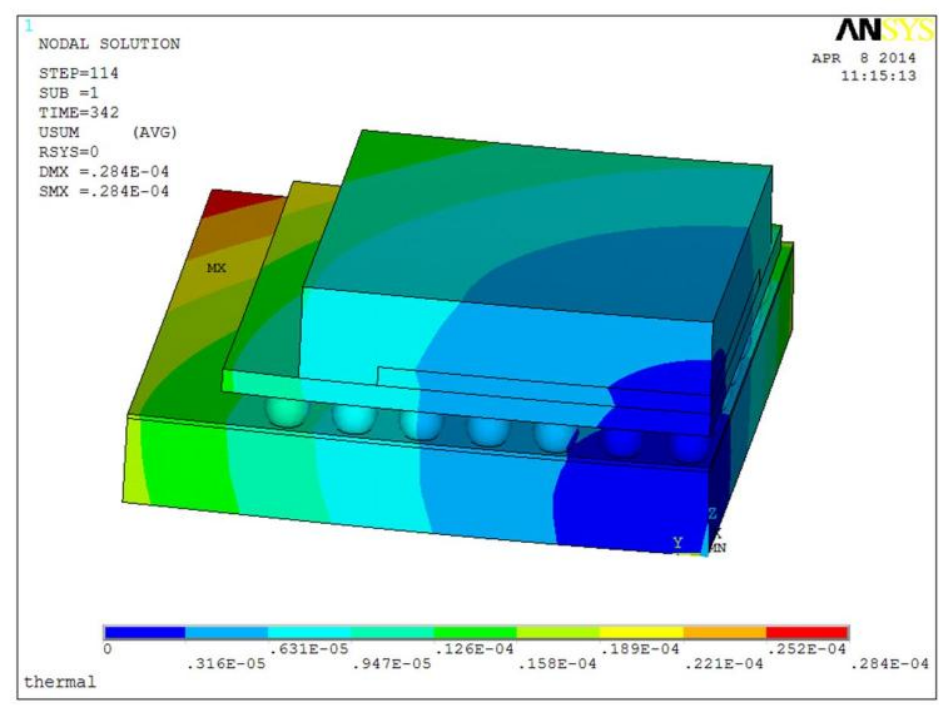

Figure 6. Strain of PCBA

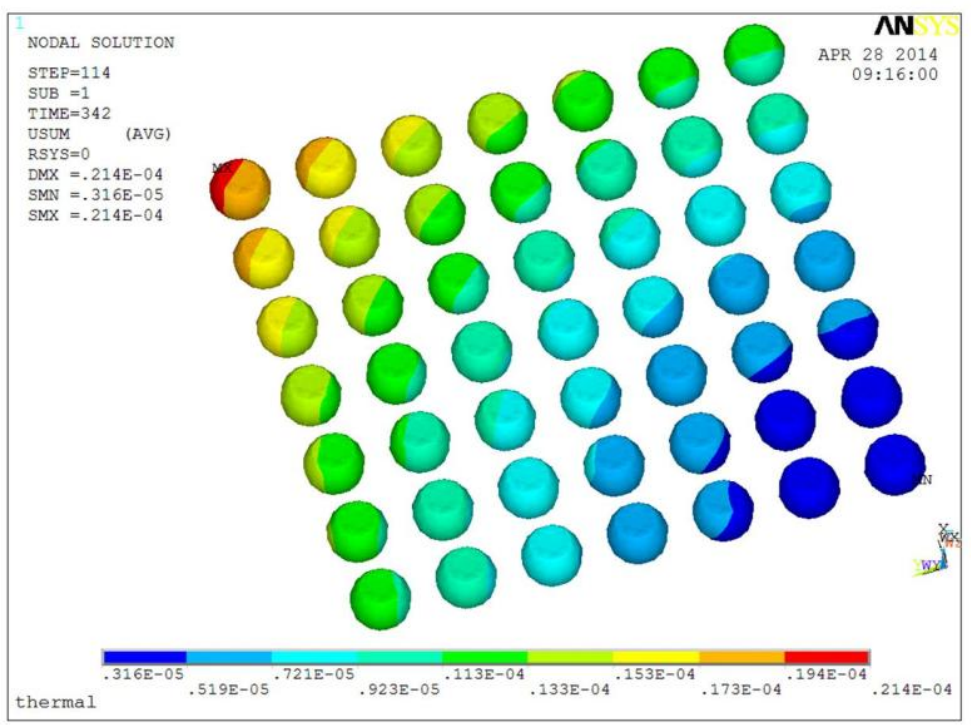

Figure 7. Strain of solder paste

From the above analysis, the maximum of thermal stress and strain appears at the corner positions of connecting surface between the solder and PBGA and BT-substrate. Meanwhile, stress and strain at the edge is greater than that in the center position. Therefore we conclude that the dangerous structural point for failure is at the corner of solder joints. Since PCBA is an axisymmetric geometry, all the four corners are dangerous structural point. When thermal stress and strain produced during the reflow process exceeds a certain value, it may lead to micro damage for solder material. These micro damages will expand and converge into cracks with thermal mechanical action in the subsequent process of using the products, which result in failure of components, interface delamination and other components failures directly. Hence, for reducing the potential failure of dangerous structural point, a preferable approach is controlling the thermal stress and strain of solder joints with improving solder material, changing the joint shape and size and adjusting the PCBA arrangements.

\section{Conclusions}

Based on the heat transfer theory, thermal stress and strain distribution of PBGA solder joint is simulated with finite element thermal-structural coupling method during the reflow process. Structural failure position of PBGA solders points is predicted as well. Study shows that thermal 
stress and strain of solder joint presents a decrease trend from the upper and lower surface to the central position respectively. At the connecting surface between the solder and PBGA and BTsubstrate, thermal stress increases gradually from the center to the surrounding of PCBA. The maximal thermal stress and strain appears at the corner positions of connecting surface between the solder and PBGA and BT-substrate. To sum up, it can be considered that the corners of solder joints are dangerous structural point.

\section{Acknowledgment}

This project is supported by the National Natural Science Foundation of China(No. 51375083) and the Fundamental Research Funds for the Central Universities of China (Grant No. N140303011).

\section{References}

1. B. Yang, Y. Liu, BGA Packaging Technology, Electronics \& Packaging, 3(4), 6-13(2003)

2. X.P. Zhang, L.M. Yin, C.B. Yu, Advances in research and application of lead-free solders for electronic and photonic packaging, Chinese Journal of Materials Research, 22(1): 1-9(2008)

3. J.E. Luan, T.E. Tee, E. Pek, Advanced numerical and experimental techniques for analysis of dynamic responses and solder joint reliability during drop impact, Components and Packaging Technologies, 29(3): 449-456(2006)

4. D.C. Whalley, A simplified reflow soldering process model, Journal of Materials Processing Technology, 150 :134-144(2004)

5. Y. Guo, Z.L. Sun., E.S. Pan and Q. Yang, Forecast of SMT Reflow Soldering Profile Based on Improved Artificial Neural Network, Journal of Northeastern University (Natural Science), 32(12):1749-1752(2011)

6. H.L. Wei, K.S. Wang, Mechanical hehavior of PBGA lead-free solder joints under cooling process of reflow soldering, Transactions of the China Welding, 33(7): 85 88(2012)

7. E. Egan, G. Kelly, T. Donovan, D. Murtagh, et al. Response Surface methodology for matrix PBGA warpage prediction, Thermal and Thermo mechanical Phenomena in Electronic System, (1): 377-384(2000)

8. Z.J. Ge, Y.X. Gu and Y.G. Jin, Thermo- structural stress analysis and optimization of PBGA package design, Journal of Dalian University of Technology, 46(9): 633 640(2006)

9. L.J.Pan, J Q.Chen. Experimental Design and Data Processing. Nanjing: Southeast University Press (2008).

10. I. Balázs, H. Gábor, Investigating direction characteristics of the heat transfer coefficient in forced convection reflow oven. Experimental Thermal and Fluid Science, 33: 642-650(2009)

11. B.Y. Huang, G.Z. Sun, T.L. Zhang, Mathematics Model of Reflow Soldering Temperature Field in SMT. Electronics Process Technology, 26(6): 333-335(2005) 